\title{
SUSY QM from Three Domain Walls in a Scalar Potential
}

\author{
R. de Lima Rodrigues* ${ }^{* \dagger}$ \\ Centro Brasileiro de Pesquisas Físicas \\ Rua Dr. Xavier Sigaud, 150 \\ CEP 22290-180, Rio de Janeiro-RJ, Brazil \\ e-mail: rafaelr@cbpf.br
}

\section{A. F. de Lima}

Unidade Acadêmica de Física, Centro de Ciências e Tecnologia, Universidade Federal de

Campina Grande, Campina Grande PB

E-mail: aerlima@df.ufcg.edu.br

\section{E. R. Bezerra de Mello and V. B. Bezerra}

Departamento de Física, Universidade Federal da Paraíba

João Pessoa-PB, 58.059-970, Brazil

e-mail: to ERBM is emello@fisica.ufph.br and to VBB is valdir@fisica.ufpb.br

\begin{abstract}
We investigate the linear classical stability of Bogomol'nyi-Prasad-Sommerfield (BPS) on three domain wall solutions in a system of three coupled real scalar fields, for a general positive potential with a square form. From a field theoretic superpotential evaluated on the domain states, the connection between the supersymmetric quantum mechanics involving three-component eigenfunctions and the stability equation associated with three classical configurations is elaborated.
\end{abstract}

Fifth International Conference on Mathematical Methods in Physics - IC2006

April 24-28 2006

Centro Brasilerio de Pesquisas Fisicas, Rio de Janeiro, Brazil

\footnotetext{
* Speaker.

†Permanent address: Unidade Acadêmica de Ciências Exatas e da Natureza, Universidade Federal de Campina Grande, Cajazeiras-PB, 58.900-000, Brazil. E-mail: rafael@df.ufcg.edu.br.
} 


\section{Introduction}

The kink solution of a field theory is an example of a soliton in $1+1$ dimensions [1, 2, 3, 4, 5]. From field theoretic superpotential [6] evaluated on the domain states, the algebraic framework of supersymmetry in quantum mechanics (SUSY QM), as formulated by Witten [7], may be elaborated. The SUSY QM generalization of the harmonic oscillator raising and lowering operators has several applications [8]. The generalization of SUSY QM for the case of matrix superpotential, is well known in the literature for a long time. See, for example, for one-dimensional systems the works in Ref. [9] about non-relativistic quantum systems, in [10, 11] about one single field and in [12, 13, 14] with two-field and in [15] on a three-field potential models in $1+1$ dimensions.

The classical configurations with domain wall solutions are bidimensional structures in $3+1$ dimensions. They are static, non-singular, classically stable Bogomol'nyi [16] and Prasad-Sommerfield [17] (BPS) soliton (defect) configurations, with finite localized energy associated with a real scalar field potential model. Domain walls have several applications in condensed matter and cosmology. The BPS states are classical configurations that satisfy the first order differential equations and the second order differential equations (equations of motion).

Recently, marginal stability and the metamorphosis of BPS states have been investigated [18], via SUSY QM, with a detailed analysis for a 2-dimensional $N=2$-Wess-Zumino model in terms of two chiral superfields, and composite dyons in $N=2$-supersymmetric gauge theories. Domain walls have been recently exploited in a context that stresses their connection with BPS-bound states. While Rajaraman has applied the trial orbit method for the equation of motion, here we use the trial orbit method for the first order differential equations associated to three real scalar fields. However, for solitons of three coupled scalar fields there are no general rules for finding analytic solutions since the nonlinearity in the potential increases the difficulties to obtain the solutions of the BPS equations and field equations.

This paper is organized as follows: In Section II, we investigate domain walls configurations for three coupled scalar fields, and supersymmetric non-relativistic quantum mechanics with threecomponent wave functions is implemented. In Section III, a scalar potential model of three coupled scalar fields is investigated. Our Conclusions are presented in Section IV.

\section{Three coupled scalar fields}

We consider the classical soliton solutions of three coupled real scalar fields in $1+1$ dimensions. They are static, nonsingular, classically stable and finite localized energy solutions of the field equations.

Recently, a superfield formulation of the central charge anomaly in quantum corrections to soliton solutions with $\mathrm{N}=1$ SUSY has been investigated [19]. The superaction in terms of the superfield and the superpotential in superspace $\left(x^{\mu} ; \Theta_{\alpha}\right), \alpha=1,2$, is written as

$$
S_{N=1}=\frac{1}{2} \int d x^{2} d^{2} \Theta\left(\sum_{i=1}^{3} \bar{D} \Phi_{i} D \Phi_{i}+W\left(\Phi_{1}, \Phi_{2}, \Phi_{3}\right)\right),
$$

where $D$ is the supercovariant derivative given by 


$$
D=\partial_{\bar{\Theta}}+i \bar{\Theta} \gamma^{\mu} \partial_{\mu}
$$

and the $\gamma^{\mu}$ are represented in terms of the Pauli matrices, $\gamma^{0}=\sigma_{y}$ and $\gamma^{1}=i \sigma_{x}$. The superpotential $W\left(\Phi_{1}, \Phi_{2}, \Phi_{3}\right)$, yields the component-field potential $V\left(\phi_{i}\right)$, where $\Phi_{i}$ are chiral superfields which, in terms of bosonic $\left(\phi_{i}\right)$, fermionic $\left(\psi_{i}\right)$ and auxiliary fields $\left(F_{i}\right)$, are $\Theta_{i}-$ expanded as shown below:

$$
\Phi_{i}=\phi_{i}+\bar{\Theta}_{i} \psi_{i}+\frac{\Theta_{i} \bar{\Theta}_{i}}{2} F_{i}
$$

where $\Theta_{i}$ and $\bar{\Theta}_{i}=\Theta_{i}^{*}$ are Grassmannian variables.

The Lagrangian density for such nonlinear system in the natural system of units $(c=\hbar=1)$, in $(1+1)$ dimensional space-time with Lorentz invariance, in terms of the bosonic fields only, is written as

$$
\mathscr{L}\left(\phi_{j}, \partial_{\mu} \phi_{j}\right)=\frac{1}{2} \sum_{j=1}^{3}\left(\partial_{\mu} \phi_{j}\right)^{2}-V\left(\phi_{j}\right)
$$

where $\partial_{\mu}=\frac{\partial}{\partial z^{\mu}}, \quad z^{\mu}=(t, z), \quad \phi_{j}=\phi_{j}(t, z), \quad(j=1,2,3)$ are real scalar fields and $\eta^{\mu v}=(+,-)$ is the metric tensor. The potential $V\left(\phi_{j}\right)=V\left(\phi_{1}, \phi_{2}, \phi_{3}\right)$ is any positive definite functional of $\phi_{j}$. The general classical configurations obey the equation bellow:

$$
\frac{\partial^{2}}{\partial t^{2}} \phi_{j}-\frac{\partial^{2}}{\partial z^{2}} \phi_{j}+\frac{\partial}{\partial \phi_{j}} V=0
$$

which, for static soliton solutions, is equivalent to the following system of nonlinear second order differential equations

$$
\phi_{j}^{\prime \prime}=\frac{\partial}{\partial \phi_{j}} V, \quad(j=1,2,3),
$$

where prime represents differentiation with respect to space variable. There is, in literature, a trial orbit method for finding static solutions for certain positive potentials, which constitutes a "trial and error" technique [2].

We can get the masses of the bosonic particles, using the results above, from the second derivatives of the potential:

$$
\left.m_{\phi_{i}}^{2} \equiv \frac{\partial^{2} V}{\partial \phi_{i}^{2}}\right|_{z \rightarrow \pm \infty}, \quad i=1,, 2,3 .
$$

\subsection{Linear Stability and SUSY QM}

Since the potential $V\left(\phi_{j}\right)$ is positive it can be written in the following square form, analogous to the case with only a single field [6],

$$
V\left(\phi_{j}\right)=V\left(\phi_{1}, \phi_{2}, \phi_{3}\right)=\frac{1}{2} \sum_{j=1}^{3} U_{j}^{2}\left(\phi_{1}, \phi_{2}, \phi_{3}\right), \quad U_{j}\left(\phi_{1}, \phi_{2}, \phi_{3}\right) \equiv \frac{\partial W}{\partial \phi_{j}}, \quad(j=1,2,3) .
$$

In this case, one can write the total energy 


$$
E=\int_{-\infty}^{+\infty} d z \frac{1}{2}\left[\left(\phi_{1}^{\prime}\right)^{2}+\left(\phi_{2}^{\prime}\right)^{2}+\left(\phi_{3}^{\prime}\right)^{2}+2 V\left(\phi_{i}\right)\right]
$$

in the BPS form, consisting of a sum of squares and surface terms,

$$
E=\int_{-\infty}^{+\infty} d z\left(\frac{1}{2}\left(\phi_{1}^{\prime}-U_{1}\right)^{2}+\frac{1}{2}\left(\phi_{1}^{\prime}-U_{2}\right)^{2}+\frac{1}{2}\left(\phi_{3}^{\prime}-U_{3}\right)^{2}+\frac{\partial}{\partial z} W\right)
$$

which are always positive. Thus, the lower bound of the energy (or classical mass) is given by the fourth term, viz.,

$$
E \geq\left|\int_{-\infty}^{+\infty} d z \frac{\partial}{\partial z} W\left[\phi_{1}(z), \phi_{2}(z), \phi_{3}(z)\right]\right|
$$

where the superpotential $W=W\left[\phi_{1}(z), \phi_{2}(z), \phi_{3}(z)\right]$ will be discussed below. The BPS mass bound of the energy resulting in a topological charge is given by

$$
E_{B P S}=T_{i j}=\left|W\left[M_{j}\right]-W\left[M_{i}\right]\right|,
$$

where $M_{i}$ and $M_{j}$ represent the BPS vacuum states and are the extrema of $W$..

In this case the BPS states satisfy the following set of first order differential equations associated to three real scalar fields:

$$
\phi_{j}^{\prime}=U_{j}\left(\phi_{1}, \phi_{2}, \phi_{3}\right)
$$

Now, let us analyze the classical stability of the soliton solutions in this nonlinear system, which can be investigated by considering small perturbations around $\phi_{1}(z), \phi_{2}(z)$ and $\phi_{3}(z)$ :

$$
\phi_{j}(t, z)=\phi_{j}(z)+\eta_{j}(t, z), \quad(j=1,2,3),
$$

where the fluctuations $\eta_{j}(t, z)$ can be expanded in terms of the normal modes as

$$
\eta_{j}(t, z)=\sum_{n} \varepsilon_{j, n} \eta_{j, n}(z) e^{i \omega_{j, n} t}, \quad \omega_{1, n}=\omega_{2, n}=\omega_{3, n}=\omega_{n}
$$

with $\varepsilon_{j, n}$ being real constant coefficients. Thus, the stability equation for the field becomes a Schrödinger-like equation of a three-component eigenfunction $\Psi_{n}$,

$$
\mathscr{H} \Psi_{n}=\omega_{n}^{2} \Psi_{n}, \quad n=0,1,2, \cdots,
$$

where

$$
\mathscr{H} \equiv-\mathbf{I} \frac{d^{2}}{d z^{2}}+V_{F}(z),
$$

with $\mathbf{I}$ being the $3 \times 3$ identity matrix. The term $V_{F}(z)$ is the $3 \times 3$ fluctuation Hessian matrix and the excited mode is given by

$$
\Psi_{n}(z)=\left(\begin{array}{c}
\eta_{1, n}(z) \\
\eta_{2, n}(z) \\
\eta_{3, n}(z)
\end{array}\right)
$$


Since

$$
V_{F i j}=\frac{\partial^{2}}{\partial \phi_{i} \partial \phi_{j}} V=\frac{\partial^{2}}{\partial \phi_{j} \partial \phi_{i}} V=V_{F j i}
$$

we see that $\mathscr{H}$ is Hermitian. Hence the eigenvalues $\omega_{n}^{2}$ of $\mathscr{H}$ are real.

The Schrödinger-like equation (2.16) and the Hessian matrix $V_{F}(z)$ are obtained by considering a Taylor expansion of the potential $V\left(\phi_{j}\right)$ in terms of $\eta_{j}(t, z)$ and retaining the first order terms in the equations of motion.

From (2.17) we find a bilinear form of $\mathscr{H}$ given by

$$
\mathscr{H}=\mathscr{A}^{+} \mathscr{A}^{-},
$$

where

$$
\mathscr{A}^{ \pm}= \pm \mathbf{I} \frac{d}{d z}+\mathbf{W}(z), \quad \mathscr{A}^{+}=\left(\mathscr{A}^{-}\right)^{\dagger}, \quad \mathbf{W}^{\dagger}(z)=\mathbf{W}(z) .
$$

Using these first order differential operators that appear in analysis of classical stability associated to a single field [6], we find

$$
\left(\mathscr{A}^{+} \mathscr{A}^{-}\right)_{j j}=-\frac{d^{2}}{d z^{2}}+\frac{\partial^{2}}{\partial \phi_{j}^{2}} V,
$$

which are exactly the diagonal element of $\mathscr{H}$. Therefore, it is easy to show that the linear stability is satisfied, which means that

$$
\omega_{n}^{2}=<\mathscr{H}>=<\mathscr{A}^{+} \mathscr{A}^{-}>=\left(\mathscr{A}^{-} \tilde{\Psi}_{n}\right)^{\dagger}\left(\mathscr{A}^{-} \tilde{\Psi}_{n}\right) \geq 0,
$$

as was stated. Also, the ground state of $\mathscr{H}$ becomes

$$
\Psi_{-}^{(0)}(z)=\left(\begin{array}{c}
U_{1}[\phi(z)] \\
U_{2}[\phi(z)] \\
U_{3}[\phi(z)]
\end{array}\right)
$$

which represents the bosonic three-component zero mode.

The 3x3-matrix superpotential satisfies the following Ricatti equation associated to the nondiagonal fluctuation Hessian $V_{F}(z)$ :

$$
\mathbf{W}^{2}+\mathbf{W}^{\prime}=V_{F}(z)=\left(\begin{array}{lll}
V_{F 11}(z) & V_{F 12}(z) & V_{F 13}(z) \\
V_{F 12}(z) & V_{F 22}(z) & V_{F 23}(z) \\
V_{F 13}(z) & V_{F 23}(z) & V_{F 33}(z)
\end{array}\right)_{\mid \phi_{i}=\phi_{i}(z)} .
$$

The Ricatti equation (2.25) holds the BPS states only. According to the Witten' SUSY model [7. 8], we have

$$
\Psi_{\operatorname{SUSY}}^{(n)}(z)=\left(\begin{array}{c}
\Psi_{-(n)}^{(n)}(z) \\
\Psi_{+}^{(n)}(z)
\end{array}\right)_{1 \mathrm{X} 6}
$$


where $\Psi_{ \pm}^{(n)}(z)$ are three-component eigenfuctions. In this case, the graded Lie algebra of the supersymmetry in quantum mechanics for the BPS states may be readily realized as

$$
\begin{gathered}
H_{S U S Y}=\left[Q_{-}, Q_{+}\right]_{+}=\left(\begin{array}{cc}
\mathscr{A}^{+} \mathscr{A}^{-} & 0 \\
0 & \mathscr{A}^{-} \mathscr{A}^{+}
\end{array}\right)_{6 \mathrm{X} 6}=\left(\begin{array}{cc}
\mathscr{H}_{-} & 0 \\
0 & \mathscr{H}_{+}
\end{array}\right), \\
{\left[H_{S U S Y}, Q_{ \pm}\right]_{-}=0=\left(Q_{-}\right)^{2}=\left(Q_{+}\right)^{2}}
\end{gathered}
$$

where $Q_{ \pm}$are the 6x6 supercharges of Witten SUSY model, viz.

$$
Q_{-}=\sigma_{-} \otimes \mathscr{A}^{-}, \quad Q_{+}=Q_{-}^{\dagger}=\left(\begin{array}{cc}
0 & \mathscr{A}^{+} \\
0 & 0
\end{array}\right)=\sigma_{+} \otimes \mathscr{A}^{+},
$$

with the intertwining operators, $\mathscr{A}^{ \pm}$, in terms of 3x3-matrix superpotential, are given by Eq. (2.26) and $\sigma_{ \pm}=\frac{1}{2}\left(\sigma_{1} \pm i \sigma_{2}\right)$, where $\sigma_{1}$ and $\sigma_{2}$ are the Pauli matrices. Of course, the bosonic sector of $H_{S U S Y}$ is exactly the fluctuating operator given by $\mathscr{H}_{-}=\mathscr{H}=-\mathbf{I} \frac{d^{2}}{d z^{2}}+\mathbf{V}_{F}(z)$, where $\mathbf{V}_{-}=\mathbf{V}_{F}(z)$ is the non-diagonal fluctuation Hessian. The supersymmetric fluctuation operator partner of $\mathscr{H}_{-}$is

$$
\mathscr{H}_{+}=\mathscr{A}^{-} \mathscr{A}^{+}=\mathscr{A}^{+} \mathscr{A}^{-}+\left[\mathscr{A}^{-}, \mathscr{A}^{+}\right]=\mathscr{H}_{-}-\mathbf{W}^{\prime}(z)
$$

so that the SUSY partner is given by $\mathbf{V}_{+}=\mathbf{V}_{-}-\mathbf{W}^{\prime}(z)$.

Starting with

$$
\mathscr{H}_{-} \Psi_{-}^{(n)}=E_{-}^{(n)} \Psi_{-}^{(n)} \Longrightarrow \mathscr{A}^{+} \mathscr{A}^{-} \Psi_{-}^{(n)}=E_{-}^{(n)} \Psi_{-}^{(n)}
$$

and multiplying (2.31) from the left by $A^{-}$we obtain

$$
\mathscr{A}^{-} \mathscr{A}^{+}\left(\mathscr{A}^{-} \Psi_{-}^{(n)}\right)=E_{-}^{(n)}\left(\mathscr{A}^{-} \Psi_{-}^{(n)}\right) \Rightarrow \mathscr{H}_{+}\left(\mathscr{A}^{-} \Psi_{-}^{(n)}\right)=E_{-}^{(n)}\left(A^{-} \Psi_{-}^{(n)}\right) .
$$

Since $\mathscr{A}^{-} \Psi_{-}^{(0)}=0$, comparison of (2.32) with

$$
\mathscr{H}_{+} \Psi_{+}^{(n)}=\mathscr{A}^{-} \mathscr{A}^{+} \Psi_{+}^{(n)}=E_{+}^{(n)} \Psi_{+}^{(n)},
$$

leads to the immediate mapping:

$$
E_{+}^{(n)}=E_{-}^{(n+1)}, \quad \Psi_{+}^{(n)} \propto A^{-} \Psi_{-}^{(n+1)}, n=0,1,2, \ldots
$$

Repeating the procedure but starting with (2.33) and multiplying the same from the left by $A^{+}$ leads to

$$
\mathscr{A}^{+} \mathscr{A}^{-}\left(\mathscr{A}^{+} \Psi_{+}^{(n)}\right)=E_{+}^{(n)}\left(\mathscr{A}^{+} \Psi_{+}^{(n)}\right)
$$

so that it follows from (2.31), (2.34) and (2.35) that

$$
\Psi_{-}^{(n+1)} \propto \mathscr{A}^{+} \Psi_{+}^{(n)}, n=0,1,2, \ldots
$$

The intertwining operator $\mathscr{A}^{-}\left(\mathscr{A}^{+}\right)$converts an eigenfunction of $\mathscr{H}_{-}\left(\mathscr{H}_{+}\right)$into an eigenfunction of $\mathscr{H}_{+}\left(\mathscr{H}_{-}\right)$with the same energy and simultaneously destroys (creates) a node of $\Psi_{-}^{(n+1)}\left(\Psi_{+}^{(n)}\right)$. 
These operations just express the content of the SUSY operations effected by $Q_{+}$and $Q_{-}$connecting the bosonic and fermionic sectors of the SUSY fluctuation operator (2.27).

The SUSY analysis presented above in fact enables the generation of a hierarchy of Hamiltonians with the eigenvalues and the eigenfunctions of the different members of the hierarchy in a simple manner. Calling $\mathscr{H}_{-}$as $\mathscr{H}_{1}$ and $\mathscr{H}_{+}$as $\mathscr{H}_{2}$, and suitably changing the subscript qualifications, and by repetition of the above procedure leads to the generation of a hierarchy of Hamiltonians given by

$$
\begin{gathered}
\mathscr{H}_{n}=-\frac{1}{2} \frac{d^{2}}{d x^{2}}+V_{n}(x)=\mathscr{A}_{n}^{+} \mathscr{A}_{n}^{-}+E_{n}^{(0)}=\mathscr{A}_{n-1}^{-} \mathscr{A}_{n-1}^{+}+E_{n-1}^{(0)}, \\
\mathscr{A}_{n}^{ \pm}= \pm \mathbf{I} \frac{d}{d z}+\mathbf{W}_{n}(z), \quad \mathbf{W}_{n}^{\dagger}(z)=\mathbf{W}_{n}(z)
\end{gathered}
$$

whose spectra satisfy the conditions

$$
\begin{aligned}
E_{1}^{n-1}= & E_{2}^{n-2}=\ldots=E_{n}^{(0)}, \quad n=2,3, \ldots, M, \\
& \Psi_{1}^{n-1} \propto \mathscr{A}_{1}^{+} \mathscr{A}_{2}^{+} \ldots \mathscr{A}_{n-1}^{+} \Psi_{n}^{(0)} .
\end{aligned}
$$

Note that the nth-member of the hierarchy has the same eigenvalue spectrum as the first member $\mathscr{H}_{1}$ except for the missing of the first $(n-1)$ eigenvalues of $\mathscr{H}_{1}$. The (n-1)th-excited state of $\mathscr{H}_{1}$ is degenerate with the ground state of $\mathscr{H}_{n}$ and can be constructed with the use of (2.40) that involves the knowledge of $A_{i}(i=1,2, \ldots, n-1)$ and $\Psi_{n}^{(0)}$.

\section{A potential model of three scalar fields}

Let us consider the following generalized potential in terms of bosonic fields only

$$
\begin{aligned}
V & =V\left(\phi_{1}, \phi_{2}, \phi_{3}\right)=\frac{1}{2}\left(\lambda \phi_{1}^{2}+\frac{\alpha}{2} \phi_{2}^{2}+\frac{\alpha}{2} \phi_{3}^{2}-\frac{m^{2}}{\lambda}\right)^{2} \\
& +\frac{1}{2}\left(-\alpha \phi_{1} \phi_{2}+\beta_{2} \phi_{3}^{2}-\beta_{2}\right)^{2} \\
& +\frac{1}{2} \phi_{3}^{2}\left(\alpha \phi_{1}-2 \beta_{2} \phi_{2}-\alpha \beta_{1}\right)^{2}
\end{aligned}
$$

where $\alpha>0$ and $\beta_{i} \geq 0$. Note that the symmetry $Z_{2} \times Z_{2}$ is only preserved if $\phi_{2}=0$ or $\beta_{1}=\beta_{2}=0$ condition is satisfied. When $\phi_{3}=0$ this potential becomes the two-field potential model recently investigated [13, 14].

From (2.6) and (3.1) the equations of motion under static limit, for each bosonic componentfield, become

$$
\begin{aligned}
\phi_{1}^{\prime \prime} & =2 \lambda \phi_{1}\left(\lambda \phi_{1}^{2}+\frac{\alpha}{2} \phi_{2}^{2}+\frac{\alpha}{2} \phi_{3}^{2}-\frac{m^{2}}{\lambda}\right) \\
& -\alpha \phi_{2}\left(\beta_{2} \phi_{3}^{2}-\alpha \phi_{2} \phi_{1}-\beta_{2}\right) \\
& -\alpha \phi_{3}^{2}\left(2 \beta_{2} \phi_{2}-\alpha \phi_{1}+\alpha \beta_{1}\right),
\end{aligned}
$$




$$
\begin{aligned}
\phi_{2}^{\prime \prime} & =\alpha \phi_{2}\left(\lambda \phi_{1}^{2}+\frac{\alpha}{2} \phi_{2}^{2}+\frac{\alpha}{2} \phi_{3}^{2}-\frac{m^{2}}{\lambda}\right) \\
& -\alpha \phi_{1}\left(\beta_{2} \phi_{3}^{2}-\alpha \phi_{1} \phi_{2}-\beta_{2}\right) \\
& -2 \beta_{2} \phi_{3}^{2}\left(-2 \beta_{2} \phi_{2}+\alpha \phi_{1}-\alpha \beta_{1}\right) \\
\phi_{3}^{\prime \prime} & =\alpha \phi_{3}\left(\lambda \phi_{1}^{2}+\frac{\alpha}{2} \phi_{2}^{2}+\frac{\alpha}{2} \phi_{3}^{2}-\frac{m^{2}}{\lambda}\right) \\
& +2 \beta_{2} \phi_{3}\left(\beta_{2} \phi_{3}^{2}-\alpha \phi_{1} \phi_{2}-\beta_{2}\right) \\
& +\phi_{3}\left(-2 \beta_{2} \phi_{2}+\alpha \phi_{1}-\alpha \beta_{1}\right)^{2} .
\end{aligned}
$$

The corresponding superpotential model in field theory is given by

$$
W\left(\phi_{j}\right)=\frac{m^{2}}{\lambda} \phi_{1}-\frac{\lambda}{3} \phi_{1}^{3}-\frac{\alpha}{2} \phi_{1} \phi_{2}^{2}-\frac{\alpha}{2} \phi_{1} \phi_{3}^{2}+\beta_{2} \phi_{2} \phi_{3}^{2}-\beta_{2} \phi_{2}+\frac{1}{2} \alpha \beta_{1} \phi_{3}^{2},
$$

where $\alpha>0$ and $\beta_{i}>0$. Thus, it is required that all $\phi_{j}, \quad j=1,2,3$ satisfy the BPS state conditions:

$$
\begin{aligned}
\phi_{1}^{\prime} & =-\left(\lambda \phi_{1}^{2}+\frac{\alpha}{2} \phi_{2}^{2}+\frac{\alpha}{2} \phi_{3}^{2}-\frac{m^{2}}{\lambda}\right) \\
\phi_{2}^{\prime} & =\beta_{2} \phi_{3}^{2}-\alpha \phi_{2} \phi_{1}-\beta_{2} \\
\phi_{3}^{\prime} & =-\phi_{3}\left(\alpha \phi_{1}-2 \beta_{2} \phi_{2}-\alpha \beta_{1}\right) .
\end{aligned}
$$

Note that the BPS states saturate the lower bound so that $E_{B P S}=\left|W_{i j}\right|$ is the central charge of the realization of $N=1$ SUSY in $1+1$ dimensions. Thus, the vacua are determined by the extrema of the superpotential

$$
\frac{\partial W}{\partial \phi_{j}}=0, \quad j=1,2,3
$$

which provides the possible vacuum states.

Let us now consider a projection on the $\left(\phi_{1}, \phi_{2}\right)$ plane in order to find an explicit form of domain walls. In this case, if we choose $\phi_{3}=0$ and $\beta_{2}=0$, the superpotential $W\left(\phi_{i}\right)$, becomes

$$
W\left(\phi_{i}\right)=\frac{m^{2}}{\lambda} \phi_{1}-\frac{\lambda}{3} \phi_{1}^{3}-\frac{\alpha}{2} \phi_{1} \phi_{2}^{2},
$$

which has been discussed recently and some orbits for this projection have been investigated in references [13, 14]. Indeed, using the vacuum states, the following trial orbit,

$$
G\left(\phi_{1}, \phi_{2}\right)=c_{1} \phi_{1}^{2}+c_{2} \phi_{2}^{2}+c_{3}=0,
$$

from $\frac{d G}{d x}=\frac{\partial G}{\partial \phi_{1}} \phi_{1}^{\prime}+\frac{\partial G}{\partial \phi_{2}} \phi_{2}^{\prime}=0, \phi_{i}^{\prime}=\frac{d \phi_{i}}{d z}$ and the BPS states (3.6), we obtain the constant coefficients, $c_{i}$. Therefore, the ground state for this projection on the $\left(\phi_{1}, \phi_{2}\right)$ plane becomes 


$$
\Psi_{-}^{(0)}(z)=\left(\begin{array}{c}
-\lambda \phi_{1}^{2}(z)-\frac{\alpha}{2} \phi_{2}^{2}(z)+\frac{m^{2}}{\lambda} \\
-\alpha \phi_{2}(z) \phi_{1}(z) \\
0
\end{array}\right)
$$

which represents the bosonic three-component zero mode. However, we have seen that if $\Psi_{-}^{(0)}$ is a normalizable three-component eigenstate, one cannot write $\Psi_{+}^{(0)}$ in terms of $\Psi_{-}^{(0)}$ in a similar manner to ordinary supersymmetric quantum mechanics. Also, $A^{-}=\psi_{-}^{(0)}\left(-\frac{d}{d x}\right) \frac{1}{\psi_{-}^{(0)}}$, is only valid for the unidimensional case with one-component eigenstate. There the superpotential is given by

$$
W(x)=\frac{d}{d x} \ln \psi_{-}^{(0)}(x) .
$$

This can not be seen in the example treated here of the classical stability analysis for three coupled real scalar fields.

The matrix superpotentials in SUSY QM for other projections of the system considered here will be investigated in a forthcoming paper, which can not be written as Eq. (3.11).

\section{Conclusion}

In this paper, we consider the classical stability analysis for BPS domain walls associated with a potential model of three coupled real scalar fields, which holds for non-ordinary supersymmetry (SUSY). The approach of effective quantum mechanics provides a realization of the SUSY algebra in the three-domain wall sector of the non-relativistic formalism.

The tensions can be deduced from the charge central properties in a model that present $N=1$ SUSY, which depend on the manifold of vacuum states, $T_{i j}=\left|W\left[M_{j}\right]-W\left[M_{i}\right]\right|$, where $M_{i}$ and $M_{j}$ represent the vacuum states.

We have shown that the positive potentials with a square form lead to three-component nonnegative normal modes $\omega_{n}{ }^{2} \geq 0$, analogous to the case with a single field [6], so that the linear stability of the Schrödinger-like equations is ensured.

A general three-component zero-mode eigenfunction is deduced. The Ricatti equation (2.25) holds the BPS states only. A detailed analysis in SUSY QM for such of a potential model will be reported separately.

In a three-field potential model there is a static classical configuration inside a topological soliton. We point out that the superpotential model investigated here can be applied in order to implement new string junctions [20] by extendeding BPS domain walls and string theory of three bosonic moduli [21]. The set of potential BPS junctions that have been identified in [22] contain the junctions that appear in [23]. Also, the BPS saturated objects with axial symmetry (wall junctions, vortices), in generalized Wess-Zumino models, have been investigated in Ref. [24].

\section{Acknowledgments}

RLR would like to acknowledge S. Alves for hospitality at CCP-CBPF of Rio de JaneiroRJ, Brazil, were the part of this work was carried out and to J. A. Helaÿel-Neto and I. V. Vancea for many stimulating discussions. This work was partially supported by Conselho Nacional de desenvolvimento Científico e Tecnológico(CNPq) and Fundação de Apoio à Pesquisa do Estado da Paraíba(FAPESQ)/CNPq(PRONEX). 


\section{References}

[1] R. Jackiw, Rev. Mod. Phys. 49 (1977) 681.

[2] R. Rajaraman, Phys. Rev. Lett., 42, 200 (1979); R. Rajaraman, Solitons and Instantons, (North-Holland, Amsterdam, 1982).

[3] S. Coleman, Aspects of Symmetry, (Cambridge University, London, 1985).

[4] A. P. Balachandran, G. Marmo, B. S. Skagerstam and A. Stern, Classical Topology and Quantum States, (World Scientific, Singapore, 1991).

[5] E. J. Weinberg, Annu. Rev. Nucl. Part. Sci. 42, 177 (1992).

[6] R. de Lima Rodrigues, Mod. Phys. Lett A10, 1309 (1995).

[7] E. Witten, Nucl. Phys. B185, 513 (1981); D. L. Pursey, Phys. Rev. D33, 2267 (1986).

[8] L. E. Gendenshtein, JETP Lett. 38, 356 (1983); L. E. Gendenshtein and I. V. Krive, Sov. Phys. Usp. 28, 645 (1985); A. Lahiri, P. K. Roy, and B. Bagchi, Int. J. Mod. Phys. A5, 1383 (1990); F. Cooper, A. Khare, and U. Sukhatme, Phys. Rep. 251, 267 (1995).

[9] A. Andrianov, F. Cannata, M. V. Ioffe and D. N. Nishnianidze J. Phys. A: Math. Gen. 30, 5037 (1997); T. Fukui, Phys. Lett. A178, 1 (1993); R. de Lima Rodrigues, "The Quantum Mechanics SUSY Algebra: an Introductory Review," Monograph CBPF MO-03/01, www.cbpf.br/biblioteca, e-print hep-th/0205017 and references therein.

[10] V. Gomes Lima, V. Silva Santos and R. de Lima Rodrigues, Phys. Lett. A298, 91 (2002), hep-th/0204175.

[11] T. Vachaspati, Phys. Rev. D69, 043510 (2004).

[12] R. de Lima Rodrigues, P. Barbosa da Silva Filho, and A. N. Vaidya, Phys. Rev. D58, 125023 (1998); R. de Lima Rodrigues, P. Barbosa da Silva Filho, and A. N. Vaidya, (1995) Proceedings of the XVI Brazilian National Meeting on Particles and Fields, pp. 446.

[13] A. Alonso Izquierdo, M. A. González León and J. Mateos Guilarte, Phys. Rev. D65, 085012 (2002), hep-th/0201200.

[14] G. de Sousa Dias, E. L. Graça and R. de Lima Rodrigues, Stability equation involving two-component eigenmodes associated to two domain walls, preprint CBPF-NF-029/02, e-print hep-th/0205195, to appear in Int. J. of Mod. Phys. A.

[15] R. de Lima Rodrigues, P. B. da Silva Filho and A. N. Vaidya, Linear classical stability from three coupled real scalar fields, XVI Brazilian National Meeting on Particles and Fields, pp. 446 (1995).

[16] E. B. Bogomol'nyi, Sov. J. Nucl. Phys. 24, 449 (1976).

[17] M. K. Prasad, C. H. Sommerfield, Phys. Rev. Lett. 35, 760 (1975).

[18] A. Ritz, M. Shifman, A. Vainshtein, and M. Voloshin, Phys. Rev., D63, 065018 (2001).

[19] K. Shizuya, Phys. Rev. D69, 065021 (2004).

[20] S. M. Carroll, S. Hellerman and M. Trodden, Phys. Rev. 61D, 065001 (2000).

[21] R. A. Battye and P. M. Sutcliffe, Phys. Rev. Lett. 86, 3989 (2001).

[22] D. Binosi and T. Veldhuis,Phys. Lett., B476, 124 (2000).

[23] P. M. Saffin, Phys. Rev. Lett. 834249 (1999).

[24] M. Shifman and T. ter Veldhuis, Phys. Rev. 62D, 065004 (2000). 